\title{
Sarcomatoid Transformation in Carcinomas: Is Precision Medicine the Answer?
}

\author{
Juwairiya Arshi', Feng Yin ${ }^{1 *}$ \\ 'Department of Pathology and Anatomical Sciences, University of Missouri, Columbia, Missouri, US
}

\section{Article Info}

\section{Article Notes}

Received: April 23, 2020

Accepted: June 01, 2020

\section{*Correspondence:}

Dr. Feng Yin, MD, PhD, Department of Pathology and Anatomical Sciences, University of Missouri, Columbia MO 65212, USA; Telephone No: (573)882-6687; Email: fengyin@health.missouri.edu.

(c) 2020 Yin F. This article is distributed under the terms of the Creative Commons Attribution 4.0 International License.

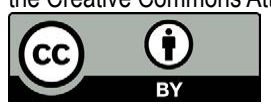

\section{Keywords:}

Sarcomatoid Transformation

Epithelial to Mesenchymal Transition

\section{ABSTRACT}

Sarcomatoid transformation in a carcinoma is a rare event but frequently associated with advanced disease stage, aggressive clinical behavior and dismal prognosis. It's likely a result of stepwise gene mutations in pluripotent stem cell and involves the epithelial to mesenchymal transition (EMT). In this review, we discuss the sarcomatoid transformation in various types of cancers. Sarcomatoid transformation in a carcinoma should always be in the differential when there is a sudden increase in size of the tumor during neoadjuvant therapy. Use of agents that interfere with the tyrosine kinase pathway might be the new potential addition to the chemotherapy regimen in these cases. Precision medicine, a rapidly emerging field, seems to be promising in the management of these cancers.

\section{Introduction}

Occurrence of biphasic tumors has been known since 1940s. However, it is being increasingly recognized that this is mainly due to the sarcomatoid transformation in cancer which involves a decrease in epithelial component and increase in mesenchymal component. This morphology is associated with distant metastases, resistance to conventional neoadjuvant therapy and dismal prognosis. Several hypotheses have been postulated to explain its biological process. However, there is increasing evidence supporting the origin of these tumors from a single pluripotent stem cell. Multiple stepwise mutations in these cells leads to sarcomatoid transformation ${ }^{1}$. Increased proliferation of mesenchymal component or increased epithelial-to-mesenchymal transition (EMT), is likely responsible for this process ${ }^{2}$.

\section{Epithelial to mesenchymal transition}

One important feature is that after the transformation, a portion of the tumor become spindle in shape and shows immunoreactivity to mesenchymal markers such as vimentin, fibronectin, and smooth muscle actin, with a decreased expression of originally epithelial cytokeratin markers ${ }^{3}$. The underlying molecular mechanisms is not entirely clear at this moment. Tumor growth factor- $\beta$ (TGF- $\beta$ ), epithelial growth factor (EGF) and insulin like growth factor (IGF) have been implicated in the sarcomatoid transformation ${ }^{4}$. The tumors upon treatment (radiotherapy and/or chemotherapy) may also acquire resistance through mutations and leads to biphasic appearance.

The sarcomatoid transformation frequently occurs when the tumor cells undergo EMT to manifest aggressive behavior and 
invasion $^{5-6}$. EMT enables the carcinoma cells to overcome physical barrier imposed by intercellular junctions ${ }^{7}$. It's also associated with an alteration in the tumor microenvironment $t^{5}$. The EMT can be induced in vitro by interfering with the tyrosine kinase signaling pathway and oncogene expression. The transformed mesenchymal cells show decreased expression of beta-catenin, E-cadherin and Alpha catenin ${ }^{5,7}$. Meanwhile the upregulation of cytoskeletal proteins confers motility and pseudopod formation and hence increased invasiveness of these cells ${ }^{7}$.

\section{Molecular alterations involved in Epithelial Mesenchymal transition}

TGF- $\beta$ is an important regulator of tumor progression. It acts as a tumor suppressor through activation of Smad mediated signaling pathway (Smad 2, Smad 3 and Smad 4). However, it could also contribute to tumorigenesis and in particular promote EMT through the mitogen-activated protein kinase (MAPK) Pathway ${ }^{8}$. Upregulation of Hic-5, a focal adhesion protein in epithelial cancer cells, is seen due to TGF- $\beta$ stimulation ${ }^{9}$. Hic- 5 is responsible for maintaining the mesenchymal phenotype with continued activation of RhoA ${ }^{9}$.

Ras, Src, Phosphatidylinositol-3 kinase (PI3K), RhoC, Rac1 and MAPK are considered as the downstream kinases implicated in the $\mathrm{EMT}^{7,10}$. These genes in turn regulate the expression of cadherin proteins. EMT is mainly driven by Ras/MAPK pathway ${ }^{10}$. Increased expression of Src was found in a study on cancer cell lines with sarcomatoid transformation $^{11}$. These changes lead to mesenchymal morphology, with increased motility and invasiveness of the tumor cells (Figure1). NF- $\kappa B$ is also implicated in the EMT pathway, though its role is poorly understood ${ }^{7,10}$. EGFR T790M mutation and MET amplification have also been implicated in the sarcomatoid transformation of lung cancer ${ }^{12}$.

\section{Sarcomatoid transformation in Head and neck cancers}

For head and neck cancers, the sarcomatoid transformation most commonly occurs in laryn $\mathrm{x}^{13-14}$ but could also be found in tongue ${ }^{15}$, gingiva ${ }^{16}$, hypopharynx, oral cavity, oropharyn $x^{17}$ and nasopharynx. ${ }^{18}$ Ionizing radiation therapy is implicated as one of the etiologic agents ${ }^{14}$. Co-expression of keratin and intermediate filaments was identified in these tumors ${ }^{19}$. The loss of heterozygosity in chromosome $17 p$ was the most remarkable finding in sarcomatoid component while both epithelial and sarcomatoid components shared other mutations. These findings support a single epithelial origin of these tumors and molecular progression responsible for this transformation ${ }^{13,20}$.

\section{Sarcomatoid transformation in Lung cancer}

Lung cancer is the leading cause of cancer related deaths worldwide. In lung cancers with typical gene mutations (EGFR and ROS1) and morphology of adenocarcinoma,

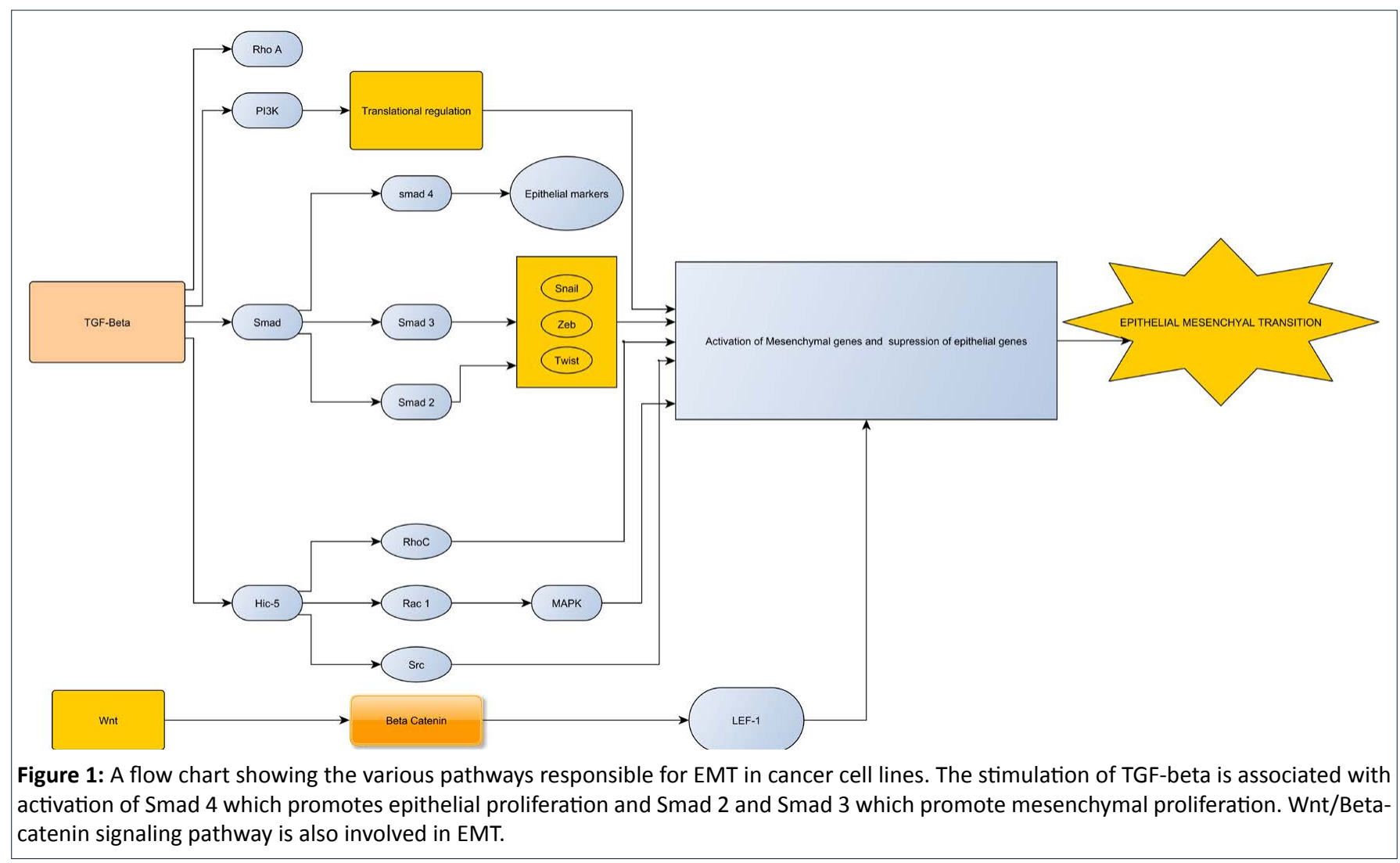


sarcomatoid transformation was observed after treatment with tyrosine kinase inhibitor after a mean interval of 31.5 months. Transformation to sarcomatoid type is present along with giant cell changes, discohesive cells, strong vimentin expression, loss of E-cadherin expression and high PDL-1 expression ${ }^{21}$. c-MET gene overexpression was also observed in 5 out of the 6 cases in this study ${ }^{21}$. This transformation might represent a resistance mechanism upon neoadjuvant therapy ${ }^{21,22}$.

There is a recent case report on a patient with an initial diagnosis of pulmonary squamous cell carcinoma. After radiotherapy, there was rapid increase in size of the tumor, and a sarcomatoid change with predominant sarcomatous component was observed. These sarcomatoid foci showed weak immunoreactivity to cytokeratin and strong immunoreactivity to vimentin ${ }^{3}$.

Mesenchymal (sarcomatoid) differentiation in mesothelioma is seen in $5-10 \%$ of cases and about twothirds of these cases are associated with distant metastases, while in typical epithelioid mesotheliomas only one third of the cases are associated with metastases ${ }^{4}$.

\section{Sarcomatoid transformation in Breast cancer}

Sarcomatoid transformation in breast cancer is a rare occurrence. The carcinosarcomas of the breast present as well circumscribed, hypoechoic mass ${ }^{23}$. These tumors are usually Her-2/neu negative and behave similar to triple negative adenocarcinomas with early metastases and overall poor survival ${ }^{24}$. With fine needle aspiration, the sarcomatoid component can be underdiagnosed due to its focality. It is always important to keep a carcinosarcoma in differential when the tumor is rapidly growing, shows early metastases and has radiologic features suggestive of a sarcomatous component.

\section{Sarcomatoid transformation in Genitourinary cancers}

Presence of sarcomatoid change is seen in 5\% of renal cancers and can be seen in up to $20 \%$ of cases with advanced disease. The sarcomatoid change is associated with extreme nuclear pleomorphism and multinucleated giant cells and is categorized by World Health Organization (WHO) as Grade IV according to WHO/ International society of Urologic Pathology grading for clear cell and papillary renal cell carcinoma ${ }^{25}$. It has also been reported in chromophobe renal cell carcinoma and is associated with poor prognosis ${ }^{26}$. Sarcomatoid renal cell cancer are usually large and associated with poor prognosis with median survival of 6 months and higher percentage of sarcomatoid components confers worse prognosis in these patients ${ }^{27}$. The hypoxia inducible pathway markers were expressed in sarcomatoid clear cell renal cell cancer, supporting a common origin of the epithelioid and sarcomatoid components. However the mechanism underlying non- clear cell sarcomatoid renal cell carcinoma appears to be different ${ }^{28,29}$. Overexpression of vascular endothelial growth factor (VEGF) was observed in majority of the clear cell and non-clear cell sarcomatoid renal cell cancers and a VEGF targeted therapy might play a promising role in treatment of these tumors ${ }^{28,29}$.

In a study done by $\mathrm{Bi}$ et al, twenty-nine cases of sarcomatoid renal cancers were selected. The carcinomatous and sarcomatoid elements were separated and whole exome sequencing was independently performed. The study revealed that the carcinomatous and sarcomatoid elements shared $41.7 \%$ of somatic single nucleotide variants (SSNVs), providing a strong evidence that both components arise from a single cell of origin that might undergo stepwise somatic mutations ${ }^{30}$. The frequency of TP53 mutations was higher in the sarcomatous component $(31.6 \%)$ when compared to that in carcinomatous components $(0 \%)^{30}$. The sarcomatoid renal cell cancers express programmed death-1 (PD-1) and programmed death ligand-1 (PDL-1) at a higher rate than their non sarcomatoid counterparts ${ }^{31}$. Expression of PD-1/ PDL-1 is also associated with higher tumor grade, necrosis and worse clinical outcomes ${ }^{32}$. Expression of these ligands may confer responsiveness to anti PD-1/ PDL-1 therapy in these tumors ${ }^{31}$. Sarcomatoid transformation has also been observed in a mature cystic teratoma of ovary ${ }^{33}$ and urinary bladder ${ }^{34,35}$.

\section{Sarcomatoid transformation in Gastrointestinal cancers}

Sarcomatoid transformation is rare in gastrointestinal tract cancers. In 1989, a study described anaplastic sarcomatoid morphology in the carcinoma of small intestine in six cases, and indicated poor prognosis with the presence of sarcomatoid morphology ${ }^{36}$. A case of duodenal sarcomatoid carcinoma showed predominant pleomorphic cells without glandular differentiation which were positive for cytokeratin and vimentin ${ }^{37}$. It has also been reported in the jejunum, where the patient presented with acute abdominal pain and small bowel perforation. This case had favorable prognosis likely due to low Ki67 proliferation index of the tumor, and the patient was recurrence free at 7 months of follow up. ${ }^{13}$ Another case with similar presentation in jejunum had an aggressive tumor showing immunoreactivity to cytokeratin, vimentin, and focal positivity for epithelial membrane antigen. Electron microscopy of the sarcomatoid component revealed irregular nuclei with prominent nucleoli, stacks of rough endoplasmic reticulum and intermediate filaments in the cytoplasm ${ }^{38-40}$. The intestinal sarcomatoid carcinoma is similar in morphology to the pleomorphic giant cell carcinomas of the lung with alveolar growth pattern and discohesive cells ${ }^{39}$. The sarcomatoid carcinoma of the small intestine is commonly present at an advanced stage with 
distant metastases ${ }^{41}$, and it is necessary to distinguish sarcomatoid tumors from other spindle cell tumors of the GI tract such as gastrointestinal stromal tumor, schwannoma and leiomyosarcoma. There are reports of sarcomatoid transformation in the hepatocellular carcinoma and combined hepatocellular cholangiocarcinoma ${ }^{42,43}$. This change could present after radiofrequency ablation ${ }^{44}$. Accumulation of mutated p53 protein might be responsible for this transformation and the associated aggressive behavior of these tumors ${ }^{45}$.

Blair et al. reported a biphasic survival and prognosis in patients with sarcomatoid transformation of pancreatic cancer. Four out of six patients had recurrence within 3 months of resection leading to death, while the other 2 patients experienced long term survival ( $>5$ years) with 16 years being the longest survival time. Notably, both patients had tumor recurrence and received radiotherapy ${ }^{46}$.

\section{Role of precision medicine}

Predictive, preventive, and personalized medicine (PPPM) is a promising and emerging field in the cancer management. PPPM, including the next generation sequencing (NGS), has provided a rapid and accurate platform for diagnosing cancer and tailoring cancer treatment to the individual characteristics of a patient ${ }^{47}$. Cancer development frequently involves specific genetic alterations. Identifying the mutations involved in sarcomatoid transformation and a personalized approach to each patient might be a better approach in treating these cancers as they are resistant to conventional chemotherapy and radiation therapies. The use of "omics", a combination of genomics, transcriptomics, proteomics, metabolomics and radiomics, with the application of NGS, high performance liquid chromatography (HPLC), Mass spectrometry (MS) and nuclear magnetic resonance technology might be the answer to the challenges faced in the treatment of these cancers ${ }^{48}$. Cancer is associated with chronic inflammation and detection of biomarkers of chronic inflammation in various types of cancer might help in prevention and early diagnosis of cancer ${ }^{49}$. Identifying the specific biomarker panel for a type of cancer through liquid biopsy and combining this with multi-omic approach might help in better patient stratification and personalized therapy for each patient ${ }^{50}$. Furthermore, a multiomic approach using machine learning may serve as an ideal tool for patient stratification and identifying the individuals at high risk ${ }^{51}$.

\section{Conclusion}

Sarcomatoid transformation could occur in many cancer types, and is associated with stepwise gene mutation leading to aggressive clinical behavior. Etiologic agents include ionizing radiation and chemotherapeutic agents, but the sarcomatoid transformation has also been reported to arise de novo. TGF- $\beta$ appears to play an important role in this transformation. The transformed cancers share many mutations with the original epithelial cancers (up to $41 \%$ ) supporting the monoclonal origin. The involvement of tyrosine kinase pathway in this transformation might lead to targeted therapy. Sarcomatoid transformation in a carcinoma should always be in the differential when there is a sudden increase in size of the tumor, and an effective treatment plan is extremely important. Use of agents that interfere with the tyrosine kinase pathway might be the new potential addition to the chemotherapy regimen in these cases. Precision medicine is a promising emerging field which might help in the treatment of these tumors and further exploration of this field in Sarcomatoid carcinomas is warranted.

\section{Conflicts of Interest}

Juwairiya Arshi, MD and Feng Yin MD, PhD have no disclosures.

\section{Funding Information}

None

\section{References}

1. Thompson L, Chang B, Barsky SH. Monoclonal Origins of Malignant Mixed Tumors (Carcinosarcomas): Evidence for a Divergent Histogenesis. Am J Surg Pathol. 1996; 20(3). https://journals.lww. com/ajsp/Fulltext/1996/03000/Monoclonal_Origins_of_Malignant_ Mixed_Tumors.3.aspx

2. Guarino M, Giordano F, Pallotti F, et al. Malignant mixed müllerian tumor of the uterus. Features favoring its origin from a common cell clone and an epithelial-to-mesenchymal transformation mechanism of histogenesis. Tumori. 1998; 84(3): 391-397.

3. Arshi J, Sauer M, Yin F. Rapid Sarcomatoid Transformation of Lung Squamous Cell Carcinoma After Neoadjuvant Therapy: A Case Report. Anticancer Res. 2020; 40(3): 1625-1629.

4. Klebanov N, Reddy BY, Husain S, et al. Cutaneous Presentation of Mesothelioma With a Sarcomatoid Transformation. Am J Dermatopathol. 2018; 40(5): 378-382.

5. Birchmeier C, Birchmeier W, Brand-Saberi B. Epithelial-Mesenchymal Transitions in Cancer Progression. Cells Tissues Organs. 1996; 156(3): 217-226.

6. Pignatelli J, Tumbarello DA, Schmidt RP, et al. Hic-5 promotes invadopodia formation and invasion during TGF- $\beta$-induced epithelial-mesenchymal transition. J Cell Biol. 2012; 197(3): 421-437.

7. Christiansen JJ, Rajasekaran AK. Reassessing Epithelial to Mesenchymal Transition as a Prerequisite for Carcinoma Invasion and Metastasis. Cancer Res. 2006; 66(17): 8319.

8. Xie L, Law BK, Chytil AM, et al. Activation of the Erk Pathway Is Required for TGF- $\beta 1$-Induced EMT In Vitro. Neoplasia. 2004; 6(5): 603-610.

9. Tumbarello DA, Turner CE. Hic-5 contributes to epithelialmesenchymal transformation through a RhoA/ROCK-dependent pathway. J Cell Physiol. 2007; 211(3): 736-747.

10. Huber MA, Kraut N, Beug H. Molecular requirements for epithelialmesenchymal transition during tumor progression. Cell--Cell Contact Extracell Matrix. 2005; 17(5): 548-558.

11. Mandal M, Myers JN, Lippman SM, et al. Epithelial to mesenchymal transition in head and neck squamous carcinoma. Cancer. 2008; 112(9): 2088-2100. 
12. Zheng J, Zhu J, Zhang K, et al. Temporal Heterogeneity of Resistance Mechanisms to EGFR-TKI Identified in a Patient With Lung Adenocarcinoma and Sarcomatoid Transformation. Clin Lung Cancer. Published online February 10, 2020.

13. Thompson LDR, Wieneke JA, Miettinen M, et al. Spindle Cell (Sarcomatoid) Carcinomas of the Larynx: A Clinicopathologic Study of 187 Cases. Am J Surg Pathol. 2002; 26(2).

14. Lambert PR, Ward PH, Berci G. Pseudosarcoma of the Larynx: A Comprehensive Analysis. Arch Otolaryngol. 1980; 106(11): 700-708.

15. Chen YK, Lin CC, Chen CH, et al. Spindle Cell Carcinoma of the Tongue : Case report and immunohisto-chemical study. Oral Med Pathol. 1998; 3(1): 51-54.

16. Katase N, Tamamura R, Gunduz M, et al. A spindle cell carcinoma presenting with osseous metaplasia in the gingiva: a case report with immunohistochemical analysis. Head Face Med. 2008; 4(1): 28.

17. Su HH, Chu ST, Hou YY, et al. Spindle Cell Carcinoma of the Oral Cavity and Oropharynx: Factors Affecting Outcome. J Chin Med Assoc. 2006; 69(10): 478-483.

18. Lee M,Son HJ,Kim NY, etal. Nasopharyngeal undifferentiated carcinoma with sarcomatoid features: Pitfalls in the immunohistochemistry. Malays J Pathol. 2019; 41(2): 201-206.

19. Meijer JWR, Ramaekers FCS, Manni JJ, et al. Intermediate filament proteins in spindle cell carcinoma of the larynx and tongue. Acta Otolaryngol (Stockh). 1988; 106(3-4): 306-313.

20. Choi HR, Sturgis EM, Rosenthal DI, et al. Sarcomatoid carcinoma of the head and neck: molecular evidence for evolution and progression from conventional squamous cell carcinomas. Am J Surg Pathol. 2003; 27(9): 1216-1220.

21. Hsieh MS, Lin MW, Lee YH. Lung adenocarcinoma with sarcomatoid transformation after tyrosine kinase inhibitor treatment and chemotherapy. Lung Cancer Amst Neth. 2019; 137: 76-84.

22. Kobayashi S, Asakura T, Ohike N, et al. Mixed acinar-endocrine carcinoma of the pancreas with intraductal growth into the main pancreatic duct: Report of a case. Surg Today. 2010; 40(4): 380-384. doi:10.1007/s00595-009-4083-9

23. Kanaizumi H, Higashi C, Tanaka Y, et al. Carcinosarcoma of the breast: A case of breast conservation surgery and breast reconstruction. SAGE Open Med Case Rep. 2019; 7: 2050313X198536842050313X19853684. doi:10.1177/2050313X19853684

24. Hennessy BT, Giordano S, Broglio K, et al. Biphasic metaplastic sarcomatoid carcinoma of the breast. Ann Oncol. 2006; 17(4): 605613. doi:10.1093/annonc/mdl006

25. Moch H, Cubilla AL, Humphrey PA, et al. The 2016 WHO Classification of Tumours of the Urinary System and Male Genital Organs-Part A: Renal, Penile, and Testicular Tumours. Eur Urol. 2016; 70(1): 93-105. doi:10.1016/j.eururo.2016.02.029

26. Bian L, Duan J, Wang X, et al. Sarcomatoid Chromophobe Renal Cell Carcinoma: A Case Report and Review of the Literature. Am J Case Rep. 2019; 20: 1225-1230. doi:10.12659/AJCR.916651

27. Mouallem NE, Smith SC, Paul AK. Sarcomatoid renal cell carcinoma: Biology and treatment advances. Urol Oncol Semin Orig Investig. 2018; 36(6): 265-271. doi:10.1016/j.urolonc.2017.12.012

28. Molina AM, Tickoo SK, Ishill N, et al. Sarcomatoid-variant renal cell carcinoma: treatment outcome and survival in advanced disease. Am J Clin Oncol. 2011; 34(5): 454-459.

29. Tickoo SK, Alden D, Olgac S, et al. Immunohistochemical Expression of Hypoxia Inducible Factor- $1 \alpha$ and its Downstream Molecules in Sarcomatoid Renal Cell Carcinoma. J Urol. 2007; 177(4): 1258-1263.

30. Bi M, Zhao S, Said JW, et al. Genomic characterization of sarcomatoid transformation in clear cell renal cell carcinoma. Proc Natl Acad Sci U

\section{S A. 2016 ; 113(8): 2170-2175.}

31. Joseph RW, Millis SZ, Carballido EM, et al. PD-1 and PD-L1 Expression in Renal Cell Carcinoma with Sarcomatoid Differentiation. Cancer Immunol Res. 2015; 3(12): 1303.

32. Choueiri TK, Fay AP, Gray KP, et al. PD-L1 expression in nonclear-cell renal cell carcinoma. Ann Oncol. 2014; 25(11): 2178-2184.

33. Pedrosa IS de S, Alpendre F da CA, Sousa R, et al. Rare Case of Sarcomatoid Squamous Cell Carcinoma Arising in an Ovarian Mature Teratoma. Rev Bras Ginecol E Obstet Rev Fed Bras Soc Ginecol E Obstet. 2019; 41(10): 628-632.

34. Yasui $\mathrm{M}$, Morikawa T, Nakagawa T, et al. Urinary bladder carcinoma with divergent differentiation featuring small cell carcinoma, sarcomatoid carcinoma, and liposarcomatous component. Pathol Res Pract. 2016; 212(9): 833-837.

35. Adachi H, Eriguchi T, Shimada S, et al. [A case report of sarcomatoid carcinoma of the urinary bladder]. Nihon Hinyokika Gakkai Zasshi Jpn J Urol. 2007; 98(3): 576-579.

36. Robey-Cafferty SS, Silva EG, Cleary KR. Anaplastic and sarcomatoid carcinoma of the small intestine: A clinicopathologic study. Hum Pathol. 1989; 20(9): 858-863.

37. Yam JL, Rai MP, Nemakayala DR, et al. Sarcomatoid carcinoma of the duodenum. BMJ Case Rep. 2018; 2018: bcr2017223991.

38. Hellquist $\mathrm{H}$, Olofsson J. Spindle cell carcinoma of the larynx. APMIS. 1989; 97(7-12): 1103-1113.

39. Bak M, Teglbjærg PS. Pleomorphic (giant cell) carcinoma of the intestine. An immunohistochemical and electron microscopic study. Cancer. 1989; 64(12): 2557-2564.

40. Battifora H. Spindle cell carcinoma. Ultrastructural evidence of squamous origin and collagen production by the tumor cells. Cancer. 1976; 37(5): 2275-2282.

41. Moriwaki Y, Sugiyama M. Severe anemia inducing preshock caused by sarcomatoid carcinoma of the small intestine. Int Surg. 2009; 94(2): 164-170.

42. Kakizoe S, Kojiro M, Nakashima T. Hepatocellular carcinoma with sarcomatous change. Clinicopathologic and immunohistochemical studies of 14 autopsy cases. Cancer. 1987; 59(2): 310-316.

43. Haratake J, Horie A. An immunohistochemical study of sarcomatoid liver carcinomas. Cancer. 1991; 68(1): 93-97.

44. Koda M, Maeda Y, Matsunaga Y, et al. Hepatocellular carcinoma with sarcomatous change arising after radiofrequency ablation for welldifferentiated hepatocellular carcinoma. Hepatol Res Off J Jpn Soc Hepatol. 2003; 27(2): 163-167.

45. Murata M, Miyoshi Y, Iwao K, et al. Combined hepatocellular/ cholangiocellular carcinoma with sarcomatoid features: genetic analysis for histogenesis. Hepatol Res Off J Jpn Soc Hepatol. 2001; 21(3): 220-227.

46. Blair $A B$, Burkhart RA, Griffin JF, et al. Long-term survival after resection of sarcomatoid carcinoma of the pancreas: an updated experience. J Surg Res. 2017; 219: 238-243.

47. Janssens JP, Schuster K, Voss A. Preventive, predictive, and personalized medicine for effective and affordable cancer care. EPMA J. 2018; 9(2): 113-123.

48. Lu M, Zhan X. The crucial role of multiomic approach in cancer research and clinically relevant outcomes. EPMA J. 2018; 9(1): 77102.

49. Qian S, Golubnitschaja O, Zhan X. Chronic inflammation: key player and biomarker-set to predict and prevent cancer development and progression based on individualized patient profiles. EPMA J. 2019; 10(4): 365-381. 
50. Golubnitschaja O, Polivka JJ, Yeghiazaryan K, et al. Liquid biopsy and multiparametric analysis in management of liver malignancies: new concepts of the patient stratification and prognostic approach. EPMA J. 2018; $9(3): 271-285$.
51. Fröhlich H, Patjoshi S, Yeghiazaryan K, et al. Premenopausal breast cancer: potential clinical utility of a multi-omics based machine learning approach for patient stratification. EPMA J. 2018; 9(2): 175186. 